\title{
Criminologie
}

\section{France 1998 : la justice des mineurs bousculée}

\section{Bruno Aubusson de Cavarlay}

Volume 32, numéro 2, automne 1999

La justice des mineurs

URI : https://id.erudit.org/iderudit/004708ar

DOI : https://doi.org/10.7202/004708ar

Aller au sommaire du numéro

Éditeur(s)

Les Presses de l'Université de Montréal

ISSN

0316-0041 (imprimé)

1492-1367 (numérique)

Découvrir la revue

\section{Citer cet article}

Aubusson de Cavarlay, B. (1999). France 1998 : la justice des mineurs bousculée. Criminologie, 32(2), 83-99. https://doi.org/10.7202/004708ar

\section{Résumé de l'article}

Depuis 1993, la question du traitement de la délinquance juvénile occupe en France une place croissante dans les discours publics et médiatiques. L'évolution des statistiques officielles alimente un sentiment d'impuissance croissant et sert d'argument aux partisans d'une réforme du droit pénal des mineurs et d'une répression nettement accrue. Cet article élargit la perspective en rappelant l'évolution de la politique judiciaire à l'égard des mineurs depuis les années 1980. La fréquence du recours à l'incarcération et le partage des cas entre l'intervention pénale (mineurs délinquants) et l'intervention civile (mineurs en danger) sont les symptômes de modifications importantes des pratiques professionnelles. La remise en cause de la législation sur les mineurs délinquants traduit la faiblesse et l'état de crise des institutions éducatives du secteur public face aux nouvelles formes de délinquance. Les mutations importantes du rapport de la justice pénale à son environnement (justice de proximité, rapidité des réponses, procédures et sanctions alternatives) donnent une place croissante à la police et au parquet. Et c'est bien au niveau des possibilités légales d'incarcération des mineurs que ces acteurs veulent imposer une sévérité plus grande.
Ce document est protégé par la loi sur le droit d'auteur. L'utilisation des services d’Érudit (y compris la reproduction) est assujettie à sa politique d'utilisation que vous pouvez consulter en ligne.

https://apropos.erudit.org/fr/usagers/politique-dutilisation/ 


\title{
France 1998 : \\ la justice des mineurs bousculée
}

\author{
Bruno Aubusson de Cavarlay \\ Chercheur \\ CNRS, Centre de recherches sociologiques \\ sur le droit et les institutions pénales. France \\ Aubusson@ext.jussieu.fr
}

RÉSUMÉ - Depuis 1993, la question du traitement de la délinquance juvénile occupe en France une place croissante dans les discours publics et médiatiques. L'évolution des statistiques officielles alimente un sentiment d'impuissance croissant et sert d'argument aux partisans d'une réforme du droit pénal des mineurs et d'une répression nettement accrue. Cet article élargit la perspective en rappelant l'évolution de la politique judiciaire à l'égard des mineurs depuis les années 1980. La fréquence du recours à l'incarcération et le partage des cas entre l'intervention pénale (mineurs délinquants) et l'intervention civile (mineurs en danger) sont les symptômes de modifications importantes des pratiques professionnelles. La remise en cause de la législation sur les mineurs délinquants traduit la faiblesse et l'état de crise des institutions éducatives du secteur public face aux nouvelles formes de délinquance. Les mutations importantes du rapport de la justice pénale à son environnement (justice de proximité, rapidité des réponses, procédures et sanctions alternatives) donnent une place croissante à la police et au parquet. Et c'est bien au niveau des possibilités légales d'incarcération des mineurs que ces acteurs veulent imposer une sévérité plus grande.

ABSTRACT - In France, since 1993, the question of how to deal with juvenile delinquency is the subject of growing public debate and media discussion. Official statistics feed a growing feeling of powerlessness and serve as an argument to these in favour of a reform of the juvenile criminal law and of increased repression. This article provides a wider perspective by reviewing the evolution of the judicial politics governing minors since the eighties. The frequency of imprisonment and the sharing of cases of criminal intervention (minor delinquents) and civil intervention (minors in danger) are signs of important modifications of the professional practice. The calling into question of juvenile delinquent legislation expresses the weakness and the state of crisis of public educational institutions vis à vis the new forms of delinquency. The important trans- 
formations which occurred in the relations between the criminal law and its environment (proximity justice, swiftness of response, alternative procedures and sanctions) give a growing place to the police and the courts. It is clear that these actors want to impose greater severity by recourse to the legal possibilities of juvenile incarceration.

Le renforcement du sentiment d'insécurité ne découle pas mécaniquement d'une augmentation de la délinquance enregistrée (Robert et Pottier, 1997). En revanche, le discours sécuritaire doit pouvoir s'alimenter de constats justifiant les appels à une répression accrue. En France, sa focalisation sur la délinquance juvénile correspond au moment où l'indicateur global de délinquance enregistrée par la police marque nettement la fin d'une croissance régulière. C'est en 1993 que s'opère ce changement de priorité : le discours officiel oppose alors la rapide montée de la délinquance juvénile à la baisse de la criminalité en général. À partir de cette année, marquée par une alternance favorable aux partis de droite, le sujet prend une priorité croissante sur l'agenda politique. Le gouvernement fait adopter des modifications législatives en tentant de satisfaire les partisans des deux camps : celui du maintien de la spécificité du droit pénal des mineurs avec le primat de l'éducatif et celui d'un retour à une répression plus systématique, adaptée à la soudaine inflation que les statistiques montreraient sans contestation possible. Quatre ans plus tard, de retour au pouvoir, la gauche doit faire face à un mouvement médiatique encore plus important. Après ce qui a été décrit comme un tournant sécuritaire marqué, quoique pâle reflet des orientations du gouvernement britannique de Tony Blair, la question du traitement de la délinquance juvénile occupe le devant de la scène.

Les premières mesures annoncées fin 1997, basées une fois de plus sur la relance des structures locales de concertation en matière de sécurité ${ }^{1}$, n'empêchent pas la pression croissante de certains acteurs, élus locaux, policiers, magistrats ou représentants des associations de victi-

1. Dans la relance de ces opérations de concertation locale, l'accent est mis sur la phase de diagnostic et sur la nécessité d'évaluer les actions, la politique de " guichet » antérieure ayant montré ses limites. Sur le plan symbolique, ces nouveaux « contrats locaux de sécurité » abandonnent le terme de « prévention » qui figurait jusque-là dans l'intitulé des programmes. Selon les partisans de ce changement, le terme de « sécurité » est plus vaste et englobe implicitement celui de « prévention». 
mes qui réclament une réforme du droit pénal des mineurs à la mesure de l'urgence de la situation. Pendant plus de six mois, la délinquance juvénile est le sujet favori des médias : toute actualité s'y rapportant, meurtre commis par un mineur ${ }^{2}$, violences scolaires, confrontations collectives de jeunes avec la police, est mise en avant ; les chaînes de télévision consacrent souvent leurs émissions documentaires et talk shows à la délinquance ou à la violence des mineurs. Dans l'urgence, le gouvernement nomme plusieurs missions traitant directement ou indirectement du sujet. Au printemps 1998, la remise des rapports et l'annonce d'arbitrages gouvernementaux sont l'occasion d'une controverse publique entre les deux principaux ministres chargés du dossier. Le ministre de l'Intérieur demande un retour aux solutions répressives seules capables de venir à bout de «sauvageons » qui menacent la République, la ministre de la Justice maintient fermement son refus d'une réforme législative remettant en cause l'idéal éducatif tout en appelant à l'innovation dans les réponses. Le premier ministre tranche en faveur de la seconde mais, dans sa sagesse, remet l'annonce publique au jour attendu en France depuis longtemps, celui de l'ouverture du Mondial. Pour quelque temps, le foot fait nettement plus recette pour les médias que la délinquance juvénile. Un répit de durée imprévisible...

Ce contexte n'a pas favorisé une réflexion sereine sur le traitement de la délinquance juvénile. L'une des spécificités françaises est sans doute d'avoir aujourd'hui, face à une situation préoccupante, un déficit considérable de travaux de recherche et surtout de recherche évaluative ${ }^{3}$. Pour ces deux raisons, il est difficile de présenter une synthèse des débats en cours sous l'éclairage des connaissances scientifiques. J'évoquerai la mise en question de la justice des mineurs selon trois axes : l'évolution de la délinquance juvénile, la place de l'État central dans son traitement et les enjeux d'une réforme du droit des mineurs.

2. Ayant été maintes fois sollicité par des journalistes pendant cette période, j'ai pris soin d'expliquer à tous mes interlocuteurs que le nombre d'homicides mis au compte de mineurs était stable depuis une dizaine d'années et en nombre suffisant pour qu'un fait nouveau soit évoqué dans les médias tous les quatre jours. Aucun à ma connaissance n'a pris le risque de reprendre ce point de vue.

3. Les travaux sur le contexte ne manquent pas (monographies à propos de quartiers sensibles, études sur la violence des jeunes, bandes...), mais pour la connaissance du fonctionnement de la justice des mineurs, les témoignages de professionnels sont le seul recours. Pour un bilan juridique et bibliographique, voir Cario (1996). 


\section{Mineurs délinquants, incivils ou sauvageons?}

Ce n'est pas en 1993 que le comportement des jeunes commence à poser des problèmes d'ordre public inédits. Leur soudaine traduction statistique à ce moment tient pour l'essentiel à une modification de la réponse pénale qui leur est apportée ${ }^{4}$. Au-delà de l'évolution du total des mineurs délinquants arrêtés par la police ${ }^{5}$, on remarquait dès le milieu des années 1980 le déplacement des traditionnelles implications pour vols de véhicules et vols à l'étalage vers des infractions plus connotées de violences, coups et blessures, destructions de biens, vols avec violence. Les mineurs ne sont pas les seuls concernés par ce mouvement mais les chiffres confirment les témoignages inquiets à propos de jeunes qui, il y a vingt ans, restaient à l'écart des formes violentes de délinquance. Les premières émeutes urbaines apparaissaient alors et impliquaient déjà des mineurs parmi les bandes qui en étaient considérées comme responsables. Mais ces «étés chauds » des banlieues étaient attribués au désœuvrement pendant les vacances scolaires des jeunes les plus défavorisés. Ils restaient vus comme des symptômes ponctuels. Aujourd'hui ils sont un aspect du vaste ensemble de la violence des jeunes dont l'explosion est le point de départ obligé de toute analyse.

Dès qu'il s'agit de préciser les choses, les catégories deviennent fuyantes. Même médiatisées à outrance ${ }^{6}$, les formes les plus graves de cette violence ne sont pas notablement plus fréquentes que par le passé. Cet argument est alors balayé par une extension sans limite des comportements visés. Ce qui est nouveau, dit-on, est le développement d'une violence banale et quotidienne, faite d'incivilités plus que de comportements pénalement qualifiables. Mais la liste qu'on donne alors en exemple comprend invariablement des infractions légalement définies et même lourdement sanctionnées. Le choix du terme d' "incivilité » par

4. Je pense en avoir démonté les principaux mécanismes dans deux articles techniques sur les statistiques policières (Aubusson de Cavarlay, 1996 et 1997). Les témoignages d'acteurs de terrain rencontrés dans le cadre de la mission interministérielle sur la prévention et le traitement de la délinquance juvénile confirment qu'à partir de 1993 les services de police sont incités à rapporter plus systématiquement les incidents connus. La hausse statistique vient alors de règles qui excluent des comptages les incidents traités en «main courante » sans signalement au parquet.

5. La statistique policière ne distingue que les mineurs de moins de 18 ans et les majeurs. Il n'est pas possible, au niveau national, de démontrer un net rajeunissement des mineurs délinquants comptabilisés.

6. Les émeutes du réveillon de Nouvel An 1998 à Strasbourg ont commencé sous l'œil des caméras d'équipes de télévision qui en avaient quasiment programmé la transmission. 
certains chercheurs (Roché, 1996) a bien permis dans un premier temps de comprendre qu'on ne pouvait restreindre l'analyse des situations d'insécurité ressentie selon les seuls critères juridico-policiers de définition de la délinquance. Mais on risque alors d'élargir inconsidérément les comportements visés et d'en diluer le contexte social pour finalement évoquer un processus de « dé-civilisation ». Le sentiment d'impuissance des acteurs de terrain trouvera ainsi une amplification sans limite dans la référence à $\mathrm{N}$. Elias et à un processus inversé de très longue durée qui pourrait s'amorcer après trois siècles de civilisation des mœurs.

Une autre façon de qualifier de nouvelles formes de délinquance juvénile consiste à parler de délinquance d'exclusion (Salas, 1997). Plus que des comportements, ce sont les caractéristiques des jeunes concernés qui sont pertinentes. Le terme d'exclusion transpose à la délinquance une notion qui place avant les différenciations traditionnelles de statut social le fait même de ne plus participer aux relations sociales normales (de famille, de travail, de quartier...). Pour les caractéristiques des mineurs ou des jeunes impliqués dans des actes de délinquance, les données empiriques sont encore absentes, mais ce type d'analyse s'appuie sur des travaux plus larges sur les jeunes « en galère ${ }^{7}$ ». Ils attestent d'une aggravation de la situation des jeunes d'origine sociale défavorisée et habitant certains quartiers. Cette aggravation prend une dimension générationnelle (exclus enfants d'exclus) et territoriale. Elle est reliée à différents indicateurs qui montrent que les jeunes sont les plus atteints par la crise économique (chômage, niveau de vie, mais aussi taux de suicide par exemple). Si elle permet une localisation plus précise des supports d'intervention publique, cette grille de lecture ne rend pas plus optimiste : on n'effacera pas sur la durée d'un programme d'intervention publique le résultat de deux générations d'urbanisme irresponsable.

Les témoignages des acteurs de terrain montrent en filigrane une unité des formes nouvelles de délinquance juvénile venant de leur immense désarroi et de l'inefficacité des réponses traditionnelles. Ce sentiment généralisé d'impuissance se retrouve chez les enseignants et chez tous les travailleurs sociaux, sans qu'il soit forcément question de délinquance. Il manifeste surtout la difficulté à faire face à des comportements collectifs ruinant les méthodes traditionnelles d'exercice de leur profession.

7. La « galère » appartient au vocabulaire des jeunes des années 1980 et a fourni le titre d'un des ouvrages de Dubet (1987). Pour une révision des rapports entre exclusion et territoire, voir Dubet (1995) ou Duprez (1996). 
Les policiers sont confrontés aux mêmes difficultés et ne supportent pas plus facilement que les autres adultes les multiples provocations auxquelles ils sont soumis dans certaines cités que l'on finit par qualifier de zones « hors droit ». D'une certaine façon, la montée de la délinquance des mineurs, comptabilisée parce que traitée sous forme de procédures judiciaires, traduit l'impossibilité dans laquelle ils se trouvent désormais de régler des incidents sans trop de gravité selon la traditionnelle voie de l'admonestation officieuse. Comme on le verra, faisant de nécessité vertu, les responsables du traitement de la délinquance juvénile s'appuient sur un consensus qui attribue tous les défauts de l'ancienne pratique à l'absence de réponse systématique aux premiers actes punissables.

\section{Le traitement de la délinquance juvénile et l'engagement de l'État}

Le primat de l'objectif éducatif affirmé par l'ordonnance de 1945, qui régit toujours la justice des mineurs, implique, lorsqu'un mineur auteur d'infraction est signalé, la recherche de mesures éducatives en priorité par rapport aux sanctions pénales. La majorité pénale est à 18 ans en France. Contrairement à ce qui est dit parfois, il n'y a pas de seuil légal inférieur de la responsabilité pénale, mais les sanctions pénales applicables varient en fonction de l'âge. En dessous de 13 ans, aucune sanction n'est possible, seules des mesures éducatives peuvent être prises par le juge des enfants saisi dans un cadre pénal. Avec des restrictions, la garde à vue lors de l'enquête de police peut être décidée dès 10 ans. De 13 à moins de 16 ans, les peines encourues sont diminuées de moitié, la détention provisoire n'est possible que pour les crimes jugés par le tribunal pour enfants. À partir de 16 ans, les principes restent les mêmes, mais l'excuse de minorité peut être écartée à titre exceptionnel; la détention provisoire peut s'appliquer en toute matière avec des durées maximum réduites; les crimes sont jugés par une cour d'assises pour mineurs.

Historiquement, ces particularités du droit des mineurs sont apparues avec l'aménagement de l'exécution des sanctions et mesures applicables en vertu d'une atténuation de la responsabilité. Dès l'origine, l'initiative privée a joué un rôle important pour créer des établissements destinés à recevoir des mineurs délinquants « acquittés » mais remis, souvent jusqu'à leur majorité, à des maisons de correction (Dupont-Bouchat et al., 1995). 
À la compétence pénale des juridictions pour mineurs est venue s'ajouter à partir de 1958 une compétence en matière civile, dite d'assistance à l'enfance en danger. En principe, ce mode d'intervention est très différent et vient compléter un dispositif administratif de protection de l'enfance. Le juge des enfants est saisi lorsque la famille refuse les mesures proposées par les services d'aide sociale à l'enfance ou lorsque les mesures envisagées enlèvent aux parents leur responsabilité juridique. L'enfant est alors considéré comme victime et normalement le juge ne devrait être saisi que par des tiers.

Cependant le juge peut se saisir lui-même d'office à titre exceptionnel. Comme les mesures décidées à titre pénal ou à titre civil sont souvent de nature comparable, il s'est établi une confusion assez grande entre les deux régimes juridiques de la justice des mineurs et la saisine civile d'office a perdu son caractère exceptionnel. L'analyse de l'enchevêtrement des deux modes de prise en charge de la jeunesse en difficulté, vocable englobant les mineurs délinquants et les mineurs en danger, est très délicate (Bailleau, 1996 : 88-102). On affirme, sans disposer de sources très précises, que la pratique $\mathrm{du}$ « double dossier » est fréquente : saisi à titre pénal ${ }^{8}$, le juge ouvre un dossier civil et prend des mesures dans ce cadre. Le dossier pénal reste en phase d'instruction ${ }^{9}$ et la décision finale peut être longtemps reportée. Cette pratique conduit à confondre le temps de la «réponse pénale » et le temps de l'observation ou de la prise en charge du mineur. Elle a été contrecarrée en 1996 par une réforme qui a donné au ministère public la possibilité de requérir une comparution à délai rapproché (entre un et trois mois). De façon générale, la question du délai de la réponse pénale aux actes de délinquance des mineurs est encore conflictuelle, alors que pour les majeurs les délais de traitement ont été considérablement réduits depuis douze ans.

Par ailleurs, le choix d'une des deux voies n'est pas sans incidence financière, les mesures éducatives prises à titre péna $1^{10}$ étant à la charge de l'État tandis que les mesures prises à titre civil sont à la charge des

8. Cette saisine pénale obéit au droit procédural général. Elle n'a lieu que sur requête du ministère public (parquet).

9. L'instruction est obligatoire pour les affaires concernant les mineurs. Le juge d'instruction compétent en toute matière peut être saisi, mais c'est le plus souvent le juge des enfants qui instruit l'affaire.

10. Le vocabulaire est ici très difficile à manier. Pour une grande partie des professionnels de la justice des mineurs, on ne peut qu'opposer mesures éducatives et sanctions pénales. La distinction entre mesures pénales (sous-entendu éducatives) et mesures civiles qui est pertinente en droit s'estompe ou disparaît dans les modalités d'application. 
collectivités locales (département). En outre, les mesures éducatives different par la nature des services qui les exécutent. Pour les mineurs délinquants, le secteur dit public (services de la Protection judiciaire de la jeunesse, PJJ) est seul compétent pour les mesures d'observation et $\mathrm{d}$ 'investigation. Destinées à préparer la décision du juge ou du tribunal des enfants, elles sont maintenant mises en œuvre par les éducateurs de services publics implantés auprès des juridictions. Le secteur public est encore seul compétent pour les mesures d'assistance éducative en milieu ouvert. Ces mesures constituant l'essentiel des décisions pénales des juridictions pour mineurs, le secteur public semble garder le monopole de la prise en charge des mineurs délinquants. Avec la pratique du « double dossier », la réalité est sans doute très différente. Pour les placements en institution, les mineurs délinquants peuvent être adressés à des établissements du secteur privé habilité. Après la fermeture des grands établissements publics pour mineurs, les placements sont devenus rares dans l'ensemble, et le secteur public n'en assure qu'une grosse moitié et même moins du tiers si l'on ne tient pas compte des placements en centre de jour propres au secteur public. Le nombre de placements dans des structures dites d'hébergement du secteur public est actuellement nettement inférieur aux incarcérations de mineurs délinquants (Aubusson de Cavarlay, 1998b).

Vu sous l'angle quantitatif, cela est la manifestation d'une crise de l'hébergement au sein du secteur public de la PJJ, relevée aussi bien par des rapports officiels (Ruffin, 1996) que par des circulaires (Justice, $1986,1993)$. Les foyers, de petite capacité, connaissent un taux de rotation du personnel éducatif important et l'accueil de mineurs délinquants particulièrement difficiles ne semble plus pouvoir y être pratiqué en même temps que l'accueil d'adolescents plus accessibles aux méthodes éducatives traditionnelles.

À partir du milieu des années 1980, une action très volontariste pour faire diminuer l'incarcération des mineurs a été suivie d'effets. C'est en renforçant les moyens d'éviter la détention provisoire des mineurs, en particulier par la systématisation des enquêtes rapides à l'issue de l'enquête policière, que ce résultat a été atteint ${ }^{11}$. Le dispositif a été accentué à partir de 1988 par une loi rendant impossible la détention provisoire des moins de 16 ans pour les délits. Le nombre de mineurs

11. Le retour de la droite au gouvernement entre 1986 et 1988 n'a pas interrompu cette évolution. 
incarcérés a décru jusqu'en 1992. Cette restriction de la ressource pénitentiaire a alors révélé l'ambiguiité du fonctionnement « en doublon » de la justice des mineurs. Il était devenu « normal » de réserver les saisines pénales aux cas de mineurs pour lesquels une incarcération pouvait être envisagée (Bailleau, 1996 : 98-99), ce qui pouvait se traduire par une détention suivie d'une peine non privative de liberté ou d'aucune peine (Tournier, 1991). Dès lors que cette voie est barrée, on observe une forte diminution des saisines pénales du juge des enfants. On peut supposer que cette baisse, qui trouve son corollaire dans la baisse de la proportion de mineurs parmi les personnes comptabilisées par la police, ne traduit pas la disparition du public qui était visé (Aubusson de Cavarlay, 1998a).

En 1993, lorsque la droite revient au pouvoir, l'impossibilité de la détention provisoire des mineurs de 16 ans demeure, mais l'absence de solution entre l'incarcération interdite et un hébergement en foyer en crise conduit les responsables politiques à envisager de nouvelles structures reposant sur un encadrement très contraignant. Le modèle des bootcamps est dans l'air, on parle beaucoup de séjours de rupture. La pression pour l'ouverture de ces structures, susceptibles d'accueillir des mineurs appartenant au « noyau dur » d'une délinquance de plus en plus violente, est surtout entretenue par les responsables policiers et répercutée par le ministre de l'Intérieur. De telles prises en charge ont été expérimentées dans le secteur privé. En 1996, parallèlement aux réformes concernant la procédure applicable aux mineurs, le ministre de la Justice tente d'imposer aux services publics l'ouverture de ces « unités à encadrement éducatif renforcé » (UEÉR). De très petite dimension (3 ou 4 mineurs, 5 éducateurs) et ne prenant en charge les mineurs que pour des durées assez courtes ( 3 mois au plus), leur nombre devait être suffisant pour répondre à une demande que le ministère évaluait à plusieurs milliers d'individus (en stocks), manifestement par excès. Le nombre de 50 unités ayant finalement été fixé, il s'est avéré que le secteur public était incapable de mobiliser les moyens nécessaires. L'opération a finalement été lancée en faisant appel conjointement au secteur privé. Pour la majorité des éducateurs de la PJJ, cette opération était le signe d'un grand tournant répressif.

En 1997, après une nouvelle alternance politique, un rapport d'évaluation était demandé au corps d'inspection administrative sur ces UEÉR dont le développement avait été suspendu. Le bilan (Gagneux et al., 1998) est assez sévère quant à la façon dont l'opération a été menée et aux possibilités du secteur public de répondre à une telle commande. 
Pour les rapporteurs, ce qui était présenté comme une réponse en grandeur nature ne pouvait être qu'une expérimentation. Seules 17 UEÉR ont été ouvertes, 14 ont pu fonctionner dont 9 dans le secteur privé. Pour les unités qui fonctionnent bien, les craintes des opposants à leur ouverture, et particulièrement celle d'ouvrir sous un nouveau nom des prisons sans réel objectif éducatif, ne se sont pas réalisées selon les rapporteurs. Cela a permis à la mission suivante (Lazerges et Balduick, 1998) de recommander le développement des structures permettant les séjours d'éloignement et de rupture, soit en UEÉR, soit dans des « lieux de vie » ou des familles d'accueil. En revanche, l'opération, menée à pas forcés, n'aura pas amélioré la mobilisation des éducateurs du secteur public pour la mise en place de solutions nouvelles de prise en charge des mineurs les plus difficiles.

Ce qu'il faut bien appeler la crise du secteur public de la PJJ repose en partie sur un cercle vicieux. Dès le début des années 1980, malgré l'abandon par la gauche des orientations sécuritaires du gouvernement précédent et le soutien des politiques de prévention, ce secteur s'est senti tenu à l'écart des évolutions (Turbelin, 1995). Le développement attendu des moyens en personnel, dont les organisations professionnelles faisaient une condition des réformes envisagées, ne s'est pas concrétisé. Les responsables politiques successifs ont quant à eux refusé jusqu'en 1998 d'attribuer massivement ces moyens alors que toutes les propositions de réformes étaient largement dénaturées par une résistance menée au nom de valeurs éducatives et humanistes fondamentales cachant de plus en plus mal un caractère corporatiste.

Le manque de moyens du secteur public est devenu évident lors des dernières missions officielles ${ }^{12}$. Entre 1986 et 1994, le nombre d'éducateurs du secteur public de la PJJ a stagné. Dans le même temps, tous les autres personnels concourant à la justice pénale ont vu leurs effectifs augmenter (Justice, 1996), avec un avantage donné aux personnels de l'administration pénitentiaire $(+45 \%$ pour les surveillants, $+32 \%$ pour les éducateurs de l'administration pénitentiaire, et $+21 \%$ de 1989 à 1994 pour les personnels des services de probation). Cet immobilisme a laissé le champ libre à d'autres acteurs.

12. Outre les deux précédents, le début de l'année 1998 a vu la multiplication des rapports de groupes de travail ou missions d'enquête sur le sujet : Chauvet (1998), Lagrange (1998), Body-Gendrot et Le Guennec (1998). 


\section{Les enjeux de l'évolution de la justice des mineurs}

Le traitement pénal de la délinquance juvénile est devenu un pan des réponses locales à l'insécurité. Au terme de cette évolution, les juges des enfants et surtout les éducateurs ont perdu leur place d'acteurs centraux de la justice des mineurs.

La première phase de décentralisation de l'intervention étatique dans les questions de délinquance et d'insécurité était orientée principalement vers la prévention. Les professionnels de la justice des mineurs pouvaient jouer leur rôle sans modifier radicalement leurs pratiques professionnelles. Leur participation aux programmes locaux de prévention était conçue autour de deux principes : assurer l'accès aux programmes généraux (formation, insertion professionnelle, loisirs) des jeunes suivis par la justice des mineurs et mettre à la disposition de ces programmes locaux les équipements dont disposait l'institution.

La prévention de la délinquance relève des collectivités locales ${ }^{13}$. Progressivement, dans le contenu des politiques publiques locales, les aspects répressifs ont pris de l'importance. L'extension de leur champ à la prévention de la récidive a permis d'associer aux programmes tous les acteurs du système pénal, y compris l'administration pénitentiaire. Dans sa version actuelle, la réponse « locale » à l'insécurité est conçue à travers une tentative de coordination de l'action d'une quantité croissante de partenaires, élus, collectivités locales, services de l'État, associations, entreprises commerciales... Leurs statuts très variés font obstacle à la collaboration. Tous les rapports officiels insistent sur ce point en indiquant qu'il reste beaucoup à faire.

Une pierre d'achoppement de cette coordination des acteurs est le refus de la Police nationale, qui a pris une place dominante dans les réponses à la délinquance juvénile, de spécialiser ses groupes d'intervention. Des brigades pour les mineurs existent encore, mais officiellement elles ne sont compétentes que pour les mineurs « victimes » ${ }^{14}$. Le motif

13. Services sous la responsabilité des maires ou des Conseils généraux, assemblées élues au niveau des départements.

14. Le débat autour de la spécialisation de forces de police pour les mineurs est rendu particulièrement confus par ce vocable de mineur « victime » qui peut signifier qu'un mineur est victime d'une infraction précise ou qu'un mineur est pris en charge au titre d'une procédure civile (mineur en danger). Avec cette seconde signification, les brigades des mineurs ont bien par le passé été compétentes pour diligenter des procédures à l'égard de mineurs auteurs d'infractions qui ne faisaient pas l'objet de poursuites pénales. En pratique, la spécialisation existait. Avec le développement des «brigades anti-criminalité » agissant essentiellement en flagrant délit, elle a été abandonnée. 
avancé est que la création d'un service spécialisé démobilise les services généraux. Tous les autres intervenants, qui connaissent une spécialisation à l'égard des mineurs, voient un danger dans ce refus. La police risque de ne plus intervenir que sur le mode du maintien de l'ordre public, ce qui implique dans les quartiers les plus difficiles un emploi croissant de la force et une escalade de l'affrontement entre jeunes et policiers. Ce mode d'action réduit d'autant les possibilités de réponses éducatives et il n'est pas non plus efficace pour enrayer la participation des mineurs à une délinquance organisée (trafic de drogue, économie souterraine). Mais ces arguments ont peu de poids face à la revendication des élus locaux d'un renforcement d'une police de proximité qui, en pratique, lorsqu'elle existe, donne la priorité à la présence visible des uniformes et aux opérations de maintien de l'ordre. Seule exception : il est prévu que les mineurs auteurs de délits commis en milieu scolaire relèveront des brigades spécialisées.

L'adaptation de la justice des mineurs semble devoir prendre acte des évolutions générales de la justice pénale. Le développement significatif des alternatives soit aux poursuites, soit à l'incarcération a modifié profondément le statut d'exception de la justice des mineurs. Les alternatives aux poursuites font bien partie des réponses de la justice pénale à des infractions signalées par la police. Elles sont mises en œuvre par le parquet dans le cadre d'une justice de «proximité » : médiation entre la victime et l'auteur, classement sous condition de régularisation ou de dédommagement, injonction thérapeutique pour les toxicomanes. C'est avec retard que ces solutions ont été appliquées à la délinquance des mineurs. Entrée dans le droit en 1993, la mesure de réparation imposée aux mineurs par le parquet est encore très inégalement utilisée, et les dernières orientations officielles en recommandent le développement (Justice, 1998). Cette capacité d'intervention du parquet sans augmenter la charge des juges pour enfants rend possible aux yeux de ses promoteurs une politique de réponse systématique aux premiers actes de délinquance commis par un mineur. Au minimum un simple « rappel à la loi », mais alors l'avertissement doit être pris au sérieux : en cas de réitération, le parquet envisagera une réponse plus marquée. Avec des appellations édulcorées et des modalités qui se veulent éducatives, on retrouve les principes en vigueur dans d'autres pays : tolérance zéro, forte gradation de la réponse avec la récidive. Dans le cas français, cette montée en force du parquet au sein de la justice des mineurs bouleverse aussi les rapports traditionnels entre les autres partenaires, policiers, juges et 
éducateurs. Les premiers se déclarent plutôt satisfaits de cette reprise en main à coloration pénale mais sont plus discrets sur le fait que les procureurs entendent par cette réorganisation rétablir leur contrôle sur la police judiciaire. Les juges et les éducateurs sont loin d'être tous opposés à ce rétablissement du parquet comme responsable de la sélection et de l'orientation des affaires pénales, y compris pour les mineurs. Ils redoutent cependant une perte de cohérence de l'intervention éducative à l'égard des mineurs délinquants. Une caractéristique traditionnelle de la justice des mineurs est d'organiser son action autour de la personnalité du mineur et de son suivi plutôt qu'en réponse cas par cas à des actes commis. Les promoteurs du traitement d'une partie de la délinquance des mineurs par le parquet, au besoin avec la mise en place de délégués du procureur ${ }^{15}$, défendent quant à eux qu'une intervention judiciaire prolongée n'est pas toujours souhaitable.

Le développement de cette «troisième voie » pose donc la question de l'intensité de la présence de la justice dans les régulations sociales (Wyvekens, 1998). Dans bien des cas, les professionnels considèrent qu'ils n'ont guère le choix et la justice pénale, pour les mineurs comme pour les majeurs, se trouve sur certains territoires, «banlieues difficiles », « quartiers sensibles », en position de seul recours pour restaurer des régulations sociales disparues. Les propositions faites à partir de cette situation, évoquant l'impossibilité ou les risques d'une judiciarisation systématique des problèmes sociaux, insistent sur la nécessité de combiner cette intervention avec une remobilisation de tous les responsables de l'éducation et de la socialisation des enfants et des adolescents. Les parents sont les premiers concernés : là encore les orientations gouvernementales refusent de renforcer les moyens coercitifs que la justice peut déjà employer en cas de démission parentale ou de complicité, voire d'incitation à la délinquance, mais en recommandent l'application. Le sujet est politiquement sensible, de nombreux élus locaux étant favorables à la suppression des aides sociales pour les parents qui ne contrôlent pas leurs enfants.

Sur ce point comme sur bien d'autres, les défenseurs de l'ordonnance de 1945 et des textes sur l'enfance en danger ont beaucoup de peine à faire admettre à ceux qui en demandent la réforme complète que leurs revendications correspondent à des textes déjà existants, peu ou mal

15. Auxiliaires de justice recrutés en fonction d'une expérience professionnelle pour exercer dans le cadre d'une justice de proximité. Cette voie d'augmentation des capacités de traitement de l'institution a déjà été mise en œuvre pour les « médiateurs ». 
appliqués. À l'ouverture d'un processus législatif qui pourrait conduire à des surenchères incontrôlables, les experts consultés ont dans leur ensemble préféré un processus qui devrait modifier profondément, voire «bousculer le fonctionnement de la justice des mineurs » (Lazerges et Balduick, 1998). Selon ces orientations, il s'agit de prendre acte du fait que l'opposition entre solution éducative et solution répressive est devenue stérile. Cela conduit, dans la circulaire de politique générale relative à la délinquance juvénile (Justice, 1998), à une reformulation de l'ambition éducative. À la « priorité donnée aux mesures éducatives » est ajoutée la « recherche de la dimension éducative dans toute sanction». Ce qui doit quand même faire frémir un certain nombre d'éducateurs...

Derrière ce glissement terminologique, c'est surtout la place de la prison qui est en jeu. En France, le système des peines appliquées aux majeurs a considérablement évolué en vingt ans. Le recours à l'incarcération a été stabilisé au milieu des années 1980, l'amende a considérablement régressé et les sanctions dites alternatives (mise à l'épreuve, travail d'intérêt général) ont pris de l'ampleur. Pour les mineurs, ces sanctions en milieu ouvert sont mises à exécution par les services de la $\mathrm{PJJ}{ }^{16}$ et, par nature, les prises en charge ne sont pas très différentes des mesures dites éducatives. D'où un effacement de l'opposition mesure éducative/sanction, d'autant plus évident que les services de milieu ouvert pour adultes ont, par leur croissance, contribué à donner une autre image de la sanction. Cela alimente sans doute en partie la « crise d'identité » de la PJJ.

Certains en viennent alors à reconsidérer le rôle de la prison et à évoquer une situation idéale où, n'étant plus une solution de facilité, elle serait une modalité d'intervention strictement réservée au cas où une mise à l'écart de la société s'impose. Très limitée en fréquence, l'incarcération serait accompagnée d'un programme individualisé de réinsertion et perdrait ainsi son rôle désocialisant. Pour les majeurs, on est très loin de cet idéal qui a alimenté en vain deux siècles de réflexions pénitentiaires. Pour les mineurs, il reste crédible, même si des aberrations comme le Centre de jeunes détenus de Fleury en région parisienne (plus de 300 détenus) donnent la pire image qui soit de la prison comme école du crime. La nécessité de prévoir des « séjours de rupture » de courte durée pour certains jeunes fait l'objet d'un consensus. La nature et le lieu de ces

16. Si le condamné est devenu majeur avant, il peut néanmoins être confié au service de probation de l'Administration pénitentiaire. Celle-ci affirme, pour le public des jeunes majeurs, une implication croissante dans tous les programmes locaux d'insertion professionnelle. 
séjours restent problématiques et en dehors de la prison, qui pour ses défenseurs est le lieu de la rupture, peu de solutions (UEÉR, lieux de vie, internat scolaire) offrent des capacités à la mesure du public concerné.

Les orientations gouvernementales récentes annoncent l'ouverture de quartiers pour mineurs de petite taille (20 places), et incitent à restaurer la continuité éducative (maintien du suivi par un éducateur PJJ) en cas d'incarcération d'un mineur. Ces orientations figuraient déjà dans les circulaires du début des années 1980 et leur inapplication a conduit à rechercher la suppression de l'incarcération des mineurs. Alors que depuis 1994 celle-ci est de nouveau en hausse, principalement en raison d'un recours accru à la détention provisoire pour les 16-17 ans, plus rien n'est dit avec vigueur pour limiter cette croissance. Un débat législatif sur la réforme de l'ordonnance de 1945 ferait probablement une ligne de clivage de cette question. Les partisans d'un retour de la possibilité de détention provisoire entre 13 et 16 ans pour les délits se sont exprimés avec insistance. Le maintien du statu quo législatif évite ce débat, mais tacitement, la croissance du nombre de mineurs incarcérés est acceptée comme une conséquence de l'évolution de la délinquance juvénile. Les orientations officielles mettent l'accent sur son traitement précoce en amont. Comme pour les majeurs, le développement des réponses alternatives est nécessaire pour éviter les situations où la prison devient inéluctable. Mais la conjoncture n'est plus celle des années 1980 et l'arrêt de la croissance de la population pénitentiaire, dont on ne connaît pas encore bien les raisons, incite moins à la vigilance que par le passé. Comme les policiers face à la stagnation des indicateurs globaux de délinquance enregistrée, les responsables de la politique pénitentiaire peuvent considérer que la réponse à la délinquance juvénile est un domaine d'investissement prioritaire, quelle que soit l'efficacité des solutions qu'ils proposent.

La justice des mineurs est donc bien bousculée. Les policiers sont les premiers acteurs du système pénal à être intervenus et continuent d'entretenir une pression dont le résultat, voulu ou non, est de tendre à la suppression de la spécificité de la réponse à l'égard des mineurs délinquants. Dans le monde judiciaire, la résistance à cette tendance est surtout le fait des acteurs qui travaillent immédiatement en aval : magistrats des parquets, mais aussi éducateurs engagés auprès d'eux dans le traitement d'urgence des cas auquel la justice pénale ne peut plus se dérober. Les nouvelles modalités d'intervention des juges spécialisés pour les mineurs sont encore incertaines. La tendance semble être de 
vouloir redonner au procès pénal sa dimension symbolique d'expression de la loi et de rétablissement des droits de chacun, cela sans évacuer pour autant la dimension éducative. L'investissement d'un nombre croissant d'avocats dans la défense des mineurs contribue à ce mouvement. Cependant, les nouvelles formes de la délinquance juvénile dite d'exclusion, très territorialisée et souvent vécue ou perçue comme contreculture répondant à un niveau intolérable d'inégalité sociale, rendent l'entreprise difficile. Les avis d'experts restent très partagés pour ce qui est de savoir jusqu'à quel point la justice peut s'engager dans des politiques coordonnées fondées sur une discrimination positive ${ }^{17}$. Tous en revanche convergent pour refuser de laisser se réduire les politiques sociales locales à une politique pénale, même habillée des vertus éducatives que le rappel à la loi semble avoir retrouvées. La force de ce refus, face à des entreprises moins réfléchies, est encore mise à rude épreuve.

\section{Références}

Aubusson De Cavarlay, B. 1996. «Les statistiques de police : méthodes de production et conditions d'interprétation ", Mathématiques, Informatique et Sciences bumaines 134 : 39-61.

AubusSON DE CAVARLAY, B. 1997. « La place des mineurs dans la délinquance enregistrée », Les Cabiers de la Sécurité Intérieure 29 : 17-38.

Aubusson DE CAVARLaY, B. 1998a. "Mesurer la délinquance juvénile», Regards sur l'actualité 238 : 41-54.

AubusSON DE CAVARLAY, B. 1998b. « Notes statistiques annexes », p. 265-288 in Réponses à la délinquance des mineurs, sous la direction de Ch. Lazerges et J.P. Balduick. Paris : La Documentation française.

BaILLEAU, F. 1996. Les jeunes face à la justice pénale. Analyse critique de l'application de l'ordonnance de 1945, Paris : Syros.

Body-Gendrot, S. et Le GuenNeC, N. 1998. Mission sur les violences urbaines, Paris : IHESI.

CARIO, R. 1996. Jeunes délinquants. À la recherche de la socialisation perdue, Paris : L'Harmattan.

CHAUVET, A. 1998. La protection de l'enfance et de la jeunesse dans un contexte social en mutation. Avis et rapport du Conseil économique et social, Paris : Les éditions des Journaux officiels.

17. Les rapports officiels n'expriment que de façon marginale le lien entre le traitement de la délinquance juvénile et les difficultés patentes d'intégration des populations issues de l'immigration. C'est par le biais d'une discrimination positive au niveau géographique (augmentation sélective des budgets locaux) qu'on tente d'aborder ce lien (Lagrange, 1998). 
DUBET, F. 1987. La galère : jeunes en survie, Paris : Fayard.

DuBET, F. 1995. «Les figures de la ville et la banlieue », Sociologie du travail 37 (2) : 127-150.

Dupont-Bouchat, M. S., Petit, J. G., Pierre, E., Schnapper, B., Tetard, F., DekKer, J., Fecteau, J. M. et TréPANier, J. 1995. Enfants corrigés, enfants protégés. Genèse de la protection de l'enfance en Belgique, en France, aux Pays-Bas et au Québec (1825-1914), Angers : HIRES.

Duprez, D. 1996. «La dramaturgie de la relégation. Vie quotidienne et sociabilité dans les cités », Les dossiers de profils, INSEE Nord Pas-de-Calais 41 : 55-73.

Gagneux, M., Feltz, F., Langlais, J. L., Vidana, J. L., Vignau, M. et Teyssier, A. 1998. Rapport sur les Unités à Encadrement Éducatif Renforcé (UEÉR) et leur apport à l'bébergement des mineurs délinquants, Paris: Inspection générale de l'administration, ronéo.

Justice (ministère de la). 1986. « Instructions relatives à l'activité des services de l'Éducation surveillée », Circulaire ES 86-98 du 5/11/86.

Justice (ministère de la). 1993. "La fonction éducative en hébergement », Circulaire PJJ 93 DIR du 30/08/93.

Justice (ministère de la). 1996. Annuaire statistique de la justice 1990-1994, Paris : La Documentation française.

Justice (ministère de la). 1998. « La politique pénale en matière de délinquance juvénile », Circulaire JUS F 9850088 C du 15/07/98.

LAGRANGE, H. 1998. Synthèse des travaux du groupe de travail sur la délinquance des mineurs sous la présidence du Garde des Sceaux, http://www.justice.gouv.fr/ publicat/lagrange.htm

LAzerges, Ch. et BALduick, J.-P. 1998. Réponses à la délinquance des mineurs, Paris : La Documentation française.

Robert, Ph. et PotTier, M. L. 1997. « "On ne se sent plus en sécurité." Délinquance et insécurité, une enquête sur deux décennies », Revue française de science politique 47 (6) : 707-740.

RochÉ, S. 1996. La société incivile. Qu'est-ce que l'insécurité?, Paris : Le Seuil.

Ruffin, M. 1996. Protection de la jeunesse et délinquance juvénile, Paris : La Documentation Française.

SALAS, D. 1997. "La délinquance d'exclusion», Les cabiers de la sécurité intérieure $29: 61-76$.

TOURNIER, P. 1991. La détention des mineurs: observation suivie d'une cohorte d'entrants, Études et données pénales nº1, Paris : CESDIP.

Turbelin, D. 1995. La justice a-t-elle besoin d'éducateurs? De l'ES à la PJ, Ramonville Saint-Agne : Érès.

WYVEKENS, A. 1998. Jeunesse en difficulté et justice de proximité, Montpellier : CEPEL. Multigraphié. 